\title{
NOUVELLE MÉTHODE D'EXTRACT1ON A FROID DE LA FRACTION LIPIDIQUE TOTALE DU JAUNE FRAIS DE L'CEUF DE POULE
}

\author{
Marie-Jeanne THLILLIE \\ avec la collaboration technique de Marie-France LOIZEAU \\ Station de Recherches avicoles. \\ Centre national de Recherches sootechniques, Jouy-en-Josas.
}

\section{SOMMAIRE}

La fraction lipidique totale d'un jaune frais peut être extraite par un mélange de : méthanolchloroforme-eau, dont les volumes sont dans les proportions $2 / 4 / 8$, en considérant le volume de l'œuf égal à I. Cette extraction se fait entièrement à froid et permet d'obtenir des lipides dont la structure peut être considérée comme intacte. Le pourcentage de récupération est : 97,72 $\pm 0,09$.

La fraction lipidique totale d'un tissu peut être extraite par différentes méthodes. Les plus utilisées jusqu'à ces dernières années font intervenir, à une étape quelconque, un ou plusieurs solvants le plus souvent à ébullition. Cependant, les méthodes à froid sont de plus en plus employées. L'on pense en général, qu'elles permettent d'obtenir des lipides dont la structure est très proche de celle qu'ils peuvent avoir dans le tissu à l'état frais.

En ce qui concerne le jaune de l'œuf de poule, RHODES et LEA (I954, I955, I957), après lyophilisation, ont extrait la fraction lipidique dans un mélange de chloroformeméthanol-eau, puis purifié par chromatographie sur colonne.

Pour des raisons d'ordre expérimental nous avons été amenés à extraire la fraction lipidique directement à partir du jaune à l'état frais.

Plusieurs auteurs ont montré, pour des tissus différents, qu'il était possible d'extraire les lipides du tissu frais avec des mélanges ternaires de : méthanol-chloroforme et eau, en proportions déterminées. Quelques essais à l'aide des quantités données par ces auteurs ne se sont pas avérés satisfaisants, la séparation de la phase chloroformique de la phase hydroalcoolique n'était jamais complète. Nous pensons que la principale cause en est que le jaune de l'œuf de poule contient beaucoup moins d'eau que les autres tissus (environ $48 \mathrm{p}$. Ioo). De plus, il renferme une quantité de lipides totaux relativement forte : 32 à $35 \mathrm{p}$. Ioo, dont un tiers environ sont des phospholipides.

Ën tenant compte de ces facteurs, nous avons défini les proportions d'un nouveau mélange de chloroforme-méthanol-eau dans lequel nous plaçons le jaune frais. 


\section{MÉ'THODE}

Io Le jaune frais est essuyé au sortir de la coquille sur un papier absorbant. Ainsi débarrassé de son blanc et des chalazes, il est ensuitepesé et rapidement introduit dans une ampoule à décanter, contenant $40 \mathrm{~cm}^{3}$ de méthanol et $80 \mathrm{~cm}^{3}$ de chloroforme.

$2^{\circ}$ Après introduction du jaune, on ajoute $r 60 \mathrm{~cm}^{3}$ d'eau distillée, on agite très fortement l'ampoule pendant une à deux minutes et on laisse les différentes phases se séparer pendant 24 heures. Le lendemain, on décante soigneusement la phase chloroformique et on ajoute $80 \mathrm{~cm}^{3}$ de chloroforme. L'ampoule est de nouveau agitée I à 2 minutes, puis laissée 24 heures.

Après décantation on ajoute ce premier lavage à l'extrait chloroformique, on lave encore deux fois avec $40 \mathrm{~cm}^{3}$ de chloroforme.

$3^{\circ}$ Après décantation des derniers $40 \mathrm{~cm}^{3}$, l'ensemble des solutions chloroformiques obtenues, pour une même ovule, est filtré dans un filtre en verre fritté $n^{0} 3$ et le filtrat ajusté à $250 \mathrm{~cm}^{3}$ avec du chloroforme.

$4^{0}$ Deux échantillons de $10 \mathrm{~cm}^{3}$ de cette solution sont évaporés, dans deux ballons tarés, à fond rond, de Ioo $\mathrm{ml}$, sous vide et sous azote. Le fond du ballon est

Récupération de la fraction lipidique totale par la méthode à froid.

\begin{tabular}{|c|c|c|c|c|c|c|c|c|}
\hline $\begin{array}{l}N^{\circ} \text { des } \\
\text { poules }\end{array}$ & $\begin{array}{c}\text { Date } \\
\text { de ponte }\end{array}$ & $\begin{array}{l}\text { Poids } \\
\text { de l'œuf } \\
\text { (g) }\end{array}$ & $\begin{array}{l}\text { Poids du } \\
\text { jaune frais } \\
\text { (g) }\end{array}$ & $\begin{array}{l}\text { Extrait } \\
\text { lipidique } \\
\text { (g) }\end{array}$ & $\begin{array}{l}\text { Résidu } \\
\text { lipidique } \\
\text { (g) }\end{array}$ & $\begin{array}{l}\text { Fraction } \\
\text { lipidique } \\
\text { totale } \\
(\mathrm{g})\end{array}$ & $\begin{array}{c}\text { Récupé- } \\
\text { ration à } \\
\text { l'extraction } \\
\text { (en p. 100) }\end{array}$ & $\begin{array}{c}\text { lipides } \\
\text { totauxpoids } \\
\text { jaune frais } \\
\text { p. } 100\end{array}$ \\
\hline 1 & $\begin{array}{c}25-9 \\
29-9 \\
30-9 \\
3-10 \\
13-10 \\
15-10 \\
20-10\end{array}$ & $\begin{array}{l}47,5014 \\
46,5016 \\
47,2016 \\
49,5688 \\
48,4348 \\
49,6406 \\
52,8303\end{array}$ & $\begin{array}{l}13,5246 \\
13,4074 \\
13,3762 \\
13,4442 \\
13,2218 \\
13,5198 \\
15,0460\end{array}$ & $\begin{array}{l}4,9250 \\
4,7525 \\
4,7650 \\
4,6375 \\
4,4225 \\
4,4375 \\
4,8537\end{array}$ & $\begin{array}{l}0,1230 \\
0,1104 \\
0,1002 \\
0,0983 \\
0,1035 \\
0,1076 \\
0,1017\end{array}$ & $\begin{array}{l}5,0480 \\
4,8629 \\
4,8652 \\
4,7358 \\
4,5260 \\
4,5451 \\
4,9554\end{array}$ & $\begin{array}{l}97,56 \\
97,73 \\
97,94 \\
97,92 \\
97,71 \\
97,63 \\
97,94\end{array}$ & $\begin{array}{l}37,32 \\
36,27 \\
36,35 \\
35,22 \\
34,23 \\
33,62 \\
32,93\end{array}$ \\
\hline 6 & $\begin{array}{l}13-10 \\
15-11 \\
18-11\end{array}$ & $\begin{array}{l}50,5972 \\
52,6633 \\
55,6181\end{array}$ & $\begin{array}{l}13,0196 \\
15,0097 \\
15,9563\end{array}$ & $\begin{array}{l}4,5025 \\
5,1600 \\
5,5325\end{array}$ & $\begin{array}{l}0,0993 \\
0,0882 \\
0,0978\end{array}$ & $\begin{array}{l}4,6018 \\
5,3482 \\
5,6303\end{array}$ & $\begin{array}{l}97,84 \\
98,35 \\
98,26\end{array}$ & $\begin{array}{l}35,35 \\
35,63 \\
35,29\end{array}$ \\
\hline 9 & $\begin{array}{l}15-11 \\
19-11\end{array}$ & $\begin{array}{r}61,7970 \\
58,7682\end{array}$ & $\begin{array}{l}16,8294 \\
15,7859\end{array}$ & $\begin{array}{l}5,8600 \\
5,5213\end{array}$ & $\begin{array}{l}0,1240 \\
0,1280\end{array}$ & $\begin{array}{l}5,9840 \\
5,6493\end{array}$ & $\begin{array}{l}97,93 \\
97,73\end{array}$ & $\begin{array}{l}35,56 \\
35,79\end{array}$ \\
\hline 10 & $\begin{array}{l}28-9 \\
29-9 \\
16-10 \\
19-10 \\
25-10\end{array}$ & $\begin{array}{l}44,3824 \\
42,4404 \\
50,9340 \\
52,8104 \\
50,44.06\end{array}$ & $\begin{array}{l}12,1220 \\
12,0994 \\
14,2262 \\
14,2768 \\
13,3558\end{array}$ & $\begin{array}{l}4,2275 \\
4,2400 \\
4,6862 \\
4,8150 \\
4,5263\end{array}$ & $\begin{array}{l}0,1142 \\
0,0938 \\
0,1221 \\
0,1073 \\
0,1155\end{array}$ & $\begin{array}{l}4,3417 \\
4,3338 \\
4,8083 \\
4,9233 \\
4,6418\end{array}$ & $\begin{array}{l}97,37 \\
97,84 \\
97,46 \\
97,82 \\
97,51\end{array}$ & $\begin{array}{l}35,82 \\
35,82 \\
33,80 \\
34,48 \\
34,75\end{array}$ \\
\hline 11 & $\begin{array}{l}27-9 \\
29-9 \\
4-10 \\
5-10 \\
7-10 \\
9-10 \\
18-10 \\
25-10\end{array}$ & $\begin{array}{l}48,6538 \\
45,9520 \\
48,3486 \\
48,9888 \\
50,3535 \\
50,6042 \\
52,6898 \\
51,1320\end{array}$ & $\begin{array}{l}12,5620 \\
12,4868 \\
12,6432 \\
12,7532 \\
13,2950 \\
13,4060 \\
14,4180 \\
14,6381\end{array}$ & $\begin{array}{l}4,3650 \\
4,3600 \\
3,9525 \\
4,3250 \\
4,5100 \\
4,5462 \\
4,9237 \\
4,8388\end{array}$ & $\begin{array}{l}0,1006 \\
0,0998 \\
0,1158 \\
0,0984 \\
0,1286 \\
0,1214 \\
0,1156 \\
0,1130\end{array}$ & $\begin{array}{l}4,4656 \\
4,4598 \\
4,0683 \\
4,4234 \\
4,6396 \\
4,6676 \\
5,0393 \\
4,9518\end{array}$ & $\begin{array}{l}97,75 \\
97,76 \\
97,15 \\
97,78 \\
97,21 \\
97-40 \\
97,71 \\
97,72\end{array}$ & $\begin{array}{l}35,55 \\
35,72 \\
32,18 \\
34,68 \\
35,08 \\
34,82 \\
34,95 \\
33,83\end{array}$ \\
\hline
\end{tabular}


légèrement réchauffé au cours de cette opération dans un courant d'air chaud. L'extrait lipidique est séché après le départ du chloroforme: pendant une demie-heure dans nos conditions expérimentales.

$5^{\circ}$ Le poids d'extrait lipidique obtenu pour Io $\mathrm{cm}^{3}$ est rapporté à $250 \mathrm{~cm}^{3}$ et donne la quantité de lipides dans le jaune d'œuf frais.

Nous avons testé cette méthode sur 25 jaunes frais provenant de 5 poules différentes.

Dans un premier temps, 1'extrait lipidique fut obtenu et calculé comme nous venons de l'indiquer. Puis, sur le résidu lipoprotéique contenu dans l'ampoule à décanter, nous avons procédé à une hydrolyse acide selon la méthode utilisée par LEROY et FRANÇOIS (I952), sur des tissus végétaux. Par cette hydrolyse nous avions un moyen d'obtenir et de doser les lipides non extraits et de mesurer aussi bien l'erreur que la fidélité de la méthode à froid.

Les résultats pour chacun des œufs étudiés sont consignés dans le tableau cijoint. I,e pourcentage de récupération est indépendant des poules dont proviennent les œufs. Le calcul statistique appliqué à ces résultats donne un pourcentage de récupération moyen de $97,72 \pm 0,09$. (erreur-type)

Reçu en aaril 1960.

\section{SUMMARY}

A TECHNIQUE TOR THE EXTRACTION OF THE TOTAL LIPID FRACTION OF FRESH EGG-YOLK aT ROOMTEMPERATURE.

The total lipid fraction of a fresh yolk can be extracted with a methanol - chloroform - water mixture, in the volume ratio of $2: 4: 8$; I representing the volume of the total egg.

The extraction can be processed completely at room temperature, preserving the original structure of the lipids. Fercentage of recuperation is $97,72 \pm 0,09$.

\section{RÉFÉRENCES BIBLIOGRAPHIQUES}

LEA C. H., Rhodes D. N., 1954. Estimation of amino-nitrogen in intact Phospholipids. Biochem. J., 56, 6I3-6I8.

LFA C. H., RHodes D. N., Stoll R. D., I955. On the chromatographic separation of glycerophospholipids. Biochem. J., 60, 353-363.

Leroy A. M., Françors A., I952. Dosage des acides gras fixes et des substances insaponifiables des tissus végétaux. Ann. Zoolech., 1, 5 I-59.

RHODES D. N., LEA A., I952. On the composition of hen's egg phospholipids. Biochen. J., 65, 52653.3 . 